\title{
Caracterização da hexaferrita de bário obtida pelo método cerâmico
}

\section{(Characterization of barium hexaferrite prepared by a ceramic synthesis method)}

\author{
R.R. Corrêa ${ }^{I}, C$.W.Pachoal ${ }^{2}$ W.A. Rocha ${ }^{1}$ \\ ${ }^{l}$ Programa de Pós-Graduação em Engenharia de Materiais, Instituto Federal de Educação, Ciência e \\ Tecnologia do Maranhão, Av. Getúlio Vargas 04, Monte Castelo, S. Luís, MA 65000-000 \\ ${ }^{2}$ Departamento de Física, Universidade Federal do Maranhão,Campus Bacanga, S. Luís, MA \\ proncor@yahoo.com.br
}

\begin{abstract}
Resumo
Este trabalho envolveu a caracterização microestrutural, vibracional e das propriedades dielétricas da hexaferrita de bário obtida pelo método cerâmico. A hexaferrita de bário $\left(\mathrm{BaFe}_{12} \mathrm{O}_{19}\right)$ foi processada por moagem de baixa energia seguida de tratamento térmico. A caracterização estrutural indicou que amostras calcinadas apresentaram a fase desejada. Entretanto, uma fase de impureza $\left(\mathrm{Ba}_{3} \mathrm{Fe}_{32} \mathrm{O}_{51}\right)$ foi observada após a moagem.

Palavras-chave: hexaferrita, caracterização, método cerâmico.
\end{abstract}

\section{Abstract}

This work involved a characterization of the structural, microstructural, vibrational and dielectric properties of barium hexaferrite prepared by a ceramic synthesis method. The barium hexaferrite $\left(\mathrm{BaFe}_{12} \mathrm{O}_{19}\right)$ was processed by low energy ball milling following by heat treatment. The structural characterization indicated that the calcined samples contained the desired phase. However, an impurity phase $\left(\mathrm{Ba}_{3} \mathrm{Fe}_{32} \mathrm{O}_{51}\right)$ was detected after milling

Keywords: hexaferrite, characterization, ceramic method.

\section{INTRODUÇÃO}

As hexaferritas de bário têm grande importância para a sociedade moderna porque são componentes de uma variedade de dispositivos eletrônicos usados em meios de gravação magnética, comunicação, geração e distribuição de energia elétrica, aplicação automotiva e equipamentos médicos, além de serem usadas como magneto em purificadores de água eliminando certas impurezas metálicas, separador de minerais paramagnéticos em minérios e direcionamento de feixes de partículas eletricamente carregadas (aceleradores de partículas).

No processamento das ferritas $\mathrm{BaM}$ pelo método cerâmico tradicional utilizam-se óxidos e carbonatos onde duas ou três etapas ocorrem para a formação da fase hexaferrita, dependendo do uso do carbonato de bário ou óxido de bário. A segunda etapa é descrita pela formação da fase intermediária monoferrita $\mathrm{BaFe}_{2} \mathrm{O}_{4}$, a qual tende a reagir com as hematitas restantes para finalmente formar a fase ferrita $\mathrm{BaM}[1,2-4]$. No processamento pelo método cerâmico utiliza-se o processo de moagem. O objetivo do processo de moagem é a diminuição do tamanho das partículas do material sólido, tendo em vista o aumento da superfície específica para melhorar a velocidade de reação, misturar de um modo mais uniforme os materiais e permitir a obtenção de um material com as características ideais de utilização [5, 6]. O rendimento da moagem é influenciado pelas características da própria matéria-prima, tais como: dimensão e forma inicial das partículas, dureza do material, resistência à compressão, ao choque, estrutura homogênea ou heterogênea, umidade ou higroscopicidade, sensibilidade à variação da temperatura e tendência à aglomeração [7].

Em sistemas como ferritas as propriedades elétricas são uma resposta a fatores tais como método de preparação, temperatura de sinterização, atmosfera de sinterização e composição química [8]. As propriedades elétricas das ferritas policristalinas também são sensíveis a microestrutura. O grão e o limite de grão são os dois principais componentes que determinam a microestrutura e conseqüentemente, as propriedades elétricas [9]. A condução nas hexaferritas é devido ao salto de elétrons entre íons $\mathrm{Fe}^{2+}$ e $\mathrm{Fe}^{3+}$. A condutividade DC nas hexaferritas aumenta quando a temperatura aumenta [10].

\section{MATERIAIS E MÉTODOS}

Os pós de óxido de ferro $\left(\mathrm{Fe}_{2} \mathrm{O}_{3}\right)$ e carbonato de bário $\left(\mathrm{BaCO}_{3}\right)$ foram homogeneizados e moídos, em moinho de 
bolas; em duas etapas, uma antes (mistura por $10 \mathrm{~min}$ ) e outra depois da calcinação (moagem propriamente dita). Os pós secos, foram prensados uniaxialmente na forma de amostras cilíndricas. As variáveis do processo de moagens utilizadas antes da calcinação foram poder de moagem (PM) 4:1 e tempo de mistura (TM) de 10 min. Em seguida os pós misturados foram calcinados a uma temperatura de $1200{ }^{\circ} \mathrm{C}$ para a obtenção da fase desejada. Após a calcinação, o pó obtido foi moído com poder de moagem de 4:1 e tempos de moagens de $5 \mathrm{~h}$ e $10 \mathrm{~h}$ para obter a diminuição do tamanho das partículas. As amostras foram tratadas termicamente à temperatura de $750{ }^{\circ} \mathrm{C}$ por $1 \mathrm{~h}$. Para caracterização elétrica foi utilizado um impedancímetro Solartron 1260 acoplado a uma interface dielétrica Solartron 1290A. As medidas de espectroscopia Raman foram realizadas em um espectrômetro Jobin-Yvon T64000 em uma configuração de retroespalhamento utilizando um laser Coherent Innova 70 operando nas linhas $514.5 \mathrm{~nm}$ como fonte de excitação. Posteriormente, foram feitas as caracterizações estruturais, microestrutural, vibracional e elétrica, das amostras.

\section{RESULTADOS E DISCUSSÃO}

As misturas dos pós dos materiais de partida $\left(\mathrm{BaCO}_{3}\right.$ e $\mathrm{Fe}_{2} \mathrm{O}_{3}$ ) foram calcinadas à temperatura de $1200^{\circ} \mathrm{C}$. Os difratogramas obtidos após calcinação são mostrados.

Os resultados mostram que após calcinação a $1200{ }^{\circ} \mathrm{C}$ por $1 \mathrm{~h}$ ocorreu transformação de fase, sendo obtida a fase de hexaferrita de bário $\mathrm{BaFe}_{12} \mathrm{O}_{19}$.

Os difratogramas dos pós obtidos com PM 4:1 e moídos 15 h são mostrados na Fig. 2.

A amostra obtida com PM 4:1 após moagem apresentou além da fase hexaferrita de bário a fase $\mathrm{Ba}_{3} \mathrm{Fe}_{32} \mathrm{O}_{51}$.

É apresentada na Fig. 3 a micrografia da amostra em pó calcinada a $1200{ }^{\circ} \mathrm{C}$ obtida com PM 4:1.

A amostra apresenta uma microestrutura com distribuição do tamanho de partículas heterogênea e com aglomerados devido ao aumento do tempo de moagem que reduziu o tamanho das partículas e aumentou a área específica. Observa-se que partículas de tamanhos menores de $200 \mathrm{~nm}$

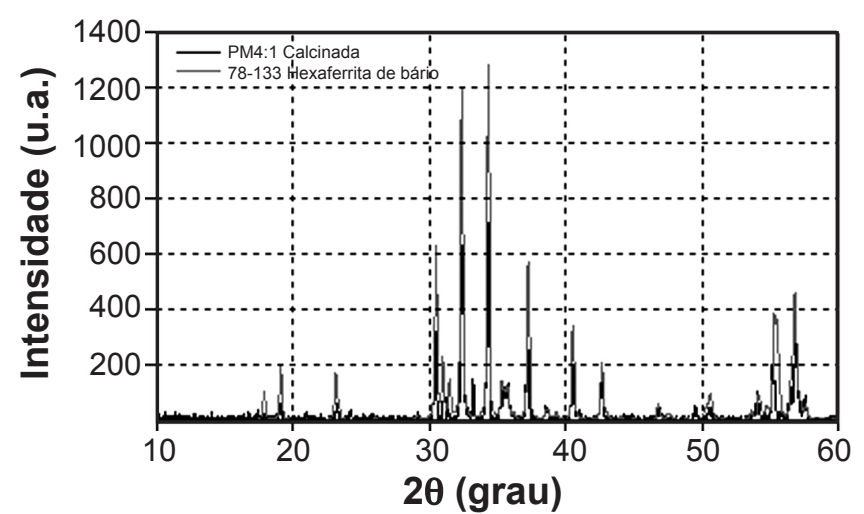

Figura 1: Difratogramas da mistura dos materiais de partida calcinadas a $1200^{\circ} \mathrm{C}$ e moída com PM 4:1.

[Figure 1: X-ray diffraction patterns of the barium ferrite sample obtained with a ball mill ratio of $4: 1$ and calcined at $\left.1200^{\circ} \mathrm{C}.\right]$

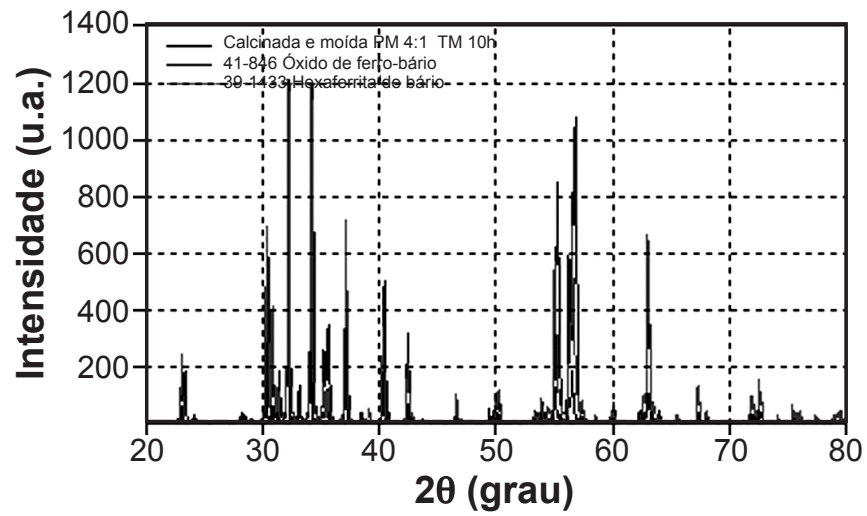

Figura 2: Difratogramas de raio X da amostra calcinada e moída com PM 4:1 e TM $10 \mathrm{~h}$.

[Figure 2: X-ray diffraction patterns of the barium ferrite sample calcined obtained with a ball mill ratio of $4: 1$ and milling time 10 h.]

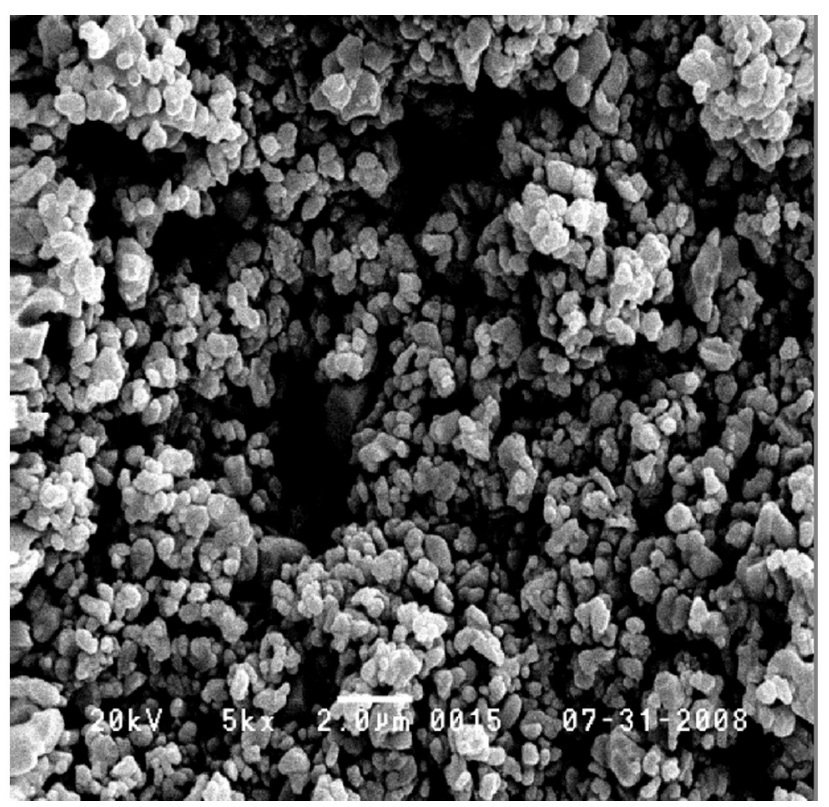

Figura 3: Micrografia da amostra obtida com PM 4:1 TM $10 \mathrm{~h}$. [Figure 3: SEM micrograph of the sample obtained with a ball mill ratio of 4:1 and after $1 \mathrm{~h}$ of ball milling.]

foram obtidas.

A Fig. 4 mostra os espectros Raman obtidos para as amostras da BaM.

O espectro é muito similar aquele obtido na literatura para a BaM, ratificando a fase magneto-plumbita. Também é possível observar que tanto o poder de moagem como o tempo de moagem não levaram a discrepâncias significativas nos espectros, o que corroborada a análise de tamanho de grão. $\mathrm{O}$ ajuste dos espectros apresentou todos os principais modos da BaM.

A Fig. 5 mostra diagramas de impedância das amostras de BaM moídas na razão de 4:1 por $10 \mathrm{~h}$ para várias temperaturas.

Para altas temperaturas a divisão entre grão e contorno de grão no espectro é mais clara. Novamente, a relaxação devido ao grão tem a frequiência incrementada quando a temperatura cresce, até desaparecer para altas temperaturas. 


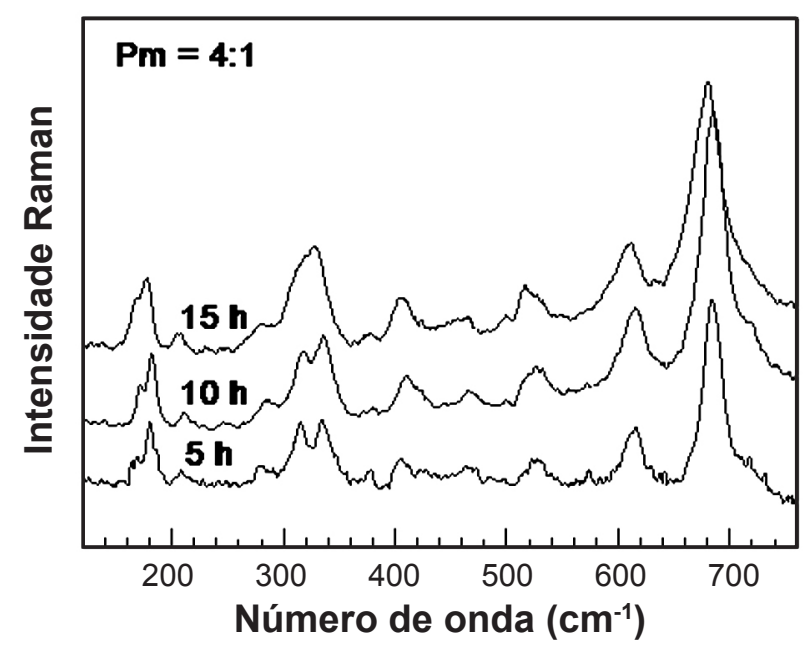

Figura 4: Espectros Raman obtidos à temperatura ambiente na ferrita BaM.

[Figure 4: Raman spectra obtained room temperature.]

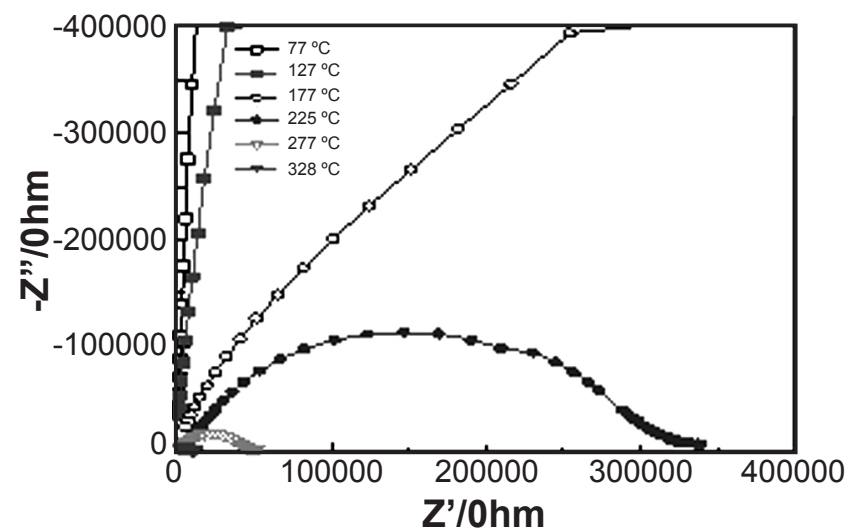

Figura 5: Diagramas de impedância da amostra de BaM moída na razão de $4: 1$ por $10 \mathrm{~h}$.

[Figure 5: Impedance plots of the barium hexaferrite samples processed with PM 4:1 ratio e and after $10 \mathrm{~h}$ of ball milling.]

As energias de ativação para os processos de relaxação, calculadas foram de $0,24 \mathrm{eV}$ e $0,68 \mathrm{eV}$ para o grão e o contorno de grão, respectivamente.

\section{CONCLUSÕES}

Foi sintetizada pelo método cerâmico e caracterizada a hexaferrita de bário. O procedimento permitiu estabelecer parâmetros, tais como poder de moagem e temperatura de calcinação, que influenciaram as características físicas e microestruturais, e que determinam um limite de propriedades que podem ser influenciadas pela microestrutura. A fase de interesse $\left(\mathrm{BaFe}_{12} \mathrm{O}_{19}\right)$ foi obtida após calcinação, embora a fase $\mathrm{Ba}_{3} \mathrm{Fe}_{32} \mathrm{O}_{51}$ tenha aparecido na microestrutura, possivelmente devido à temperatura de calcinação e composição estabelecida na estequiometria. Os espectros Raman obtidos à temperatura ambiente foram praticamente os mesmo para todas as amostras, confirmando a fase magneto-plumbita como fase dominante, sendo os modos mais importantes observados. A relaxação associada à transferência de elétrons entre os íons $\mathrm{Fe}^{2+}$ e $\mathrm{Fe}^{3+}$ foi observada nas perdas através de uma para frequiências da ordem de $100 \mathrm{kHz}$. Para altas temperaturas foi possível distinguir entre os arcos devidos ao grão e ao contorno de grão. Foi observado que a contribuição de contorno de grão diminuiu consideravelmente à medida que a temperatura aumentou. Com isso, foi possível separar as contribuições dos grãos e dos contornos de grão. A resistência do grão diminui com o aumento da temperatura, mostrando o caráter semicondutor da hexaferrita de bário. Para $\mathrm{PM}=4: 1$, a resistência elétrica decresce exponencialmente com uma energia de ativação de $0,28 \mathrm{eV}$, enquanto que a resistência dos contornos de grão diminui com energia de ativação de $\sim 0,79 \mathrm{eV}$.

\section{AGRADECIMENTOS}

Ao CNPq, ao IFMA e a UFMA pelo incentivo à pesquisa

\section{REFERÊNCIAS}

[1] K. S. Martirosyan, D. Litvinov, Barium hexaferrite nanoparticles: synthesis and magnetic properties, Mater. Sci. Eng. B 176 (2011) 8-13.

[2] G. Benito, J. S. Moya, Barium hexaferrite monodispersed nanoparticles prepared by the ceramic method, J. Magn. Magn. Mater. 234 (2001) 65-72.

[3] P. Sharma, R. A. Rocha, Structural and magnetic studies on barium hexaferrites prepared by mechanical alloying and conventional route, J. Alloys Compd. 443 (2007) 37-42. [4] K. Mallick, P. Shepherd, J. R. Green, Dielectric properties of M-type barium hexaferrite prepared by co-precipitation, J. Eur. Ceram. Soc. 27 (2007) 2045-2052.

[5] C. Suryanarayana, The science and technology of mechanical alloying, Mater. Sci. Eng. A 304-306 (2001) 151-158.

[6] W. A. Kaczmare, S. J. Campbell, M. Schmidt, Structural investigation of milled $\alpha-F e$ and barium ferrite nanomixture, Mater. Sci. Forum 312-314 (1999) 197.

[7] A. J. O. Cabral, T. Ogasawara,L. M. Tavares, "Preparação de Óxido de Ferro Micrométrico para Aplicação em Cerâmicas Magnéticas por Moagem de Alta Energia", Anais 48o Cong. Bras. Ceram., Curitiba, PR (2004).

[8] M. A. EL Hiti, A. M. EL Ata, Semiconductivity in $\mathrm{Ba}_{2} \mathrm{~N}_{i 2}$ $\mathrm{Zn}_{x} \mathrm{Fe}_{12} \mathrm{O}_{22}$ y type hexaferrites, J. Magn. Magn. Mater. 195 (1999) 667-678.

[9] M. H. Abdullah, A. N. Yusoff, Complex impedance and dielectric properties of an $\mathrm{Mg}$ - Zn ferrite, J. Alloy. Compd. 233 (1996) 129-135.

[10] A. M. Abo, E. Ata, M. K. Nimr, Conduction mechanism of $\mathrm{BaCO}_{2-x} \mathrm{Ni}_{x} \mathrm{Fe}_{16} \mathrm{O}_{27}$, J. Magn. Magn. Mater. 202 (1999) 397-404.

(Rec. 16/07/2012, Ac. 22/04/2013) 